\title{
Optics
}

\section{Size quantization Stark effect in quantum dots}

\author{
Sergey I. Pokutnyi, Oksana V. Naumenko \\ Chuiko Institute of Surface Chemistry, National Academy of Sciences of Ukraine, Kyiv, Ukraine
}

Email address:

pokutnyi_sergey@inbox.ru (S. I. Pokutnyi)

\section{To cite this article:}

Sergey I. Pokutnyi, Oksana V. Naumenko. Size Quantization Stark Effect in Quantum Dots. Optics. Special Issue: Optics and Spectroscopy of the Charge Carriers and Excitons States in Quasi - Zero - Dimensional Nanostructures. Vol. 3, No. 6-1, 2014, pp. 57-60. doi: $10.11648 /$ j.optics.s.2014030601.19

\begin{abstract}
Advantages of semiconductor quantum dots for study and diagnostics of biological nanosystems are discussed. A new method for amino acid diagnostics used semiconductor quantum dots is posed. Interaction of isolated quantum dots with charged amino acids is studied in detail. It is shown that such interaction results in a shift of the quantum dots luminescence spectra by several dozens of meV. This effect provides new possibilities for identification of biological nanoobjects using quantum dots.
\end{abstract}

Keywords: Size Quantization Stark Effect, Biological Nanosystems, Quantum Dots, Energy Shift

\section{Introduction}

Currently intensively studied electro-optical properties of various quasi-zero-semiconductor structures with spherical symmetry - such as quantum dots (QD) [1-15], and also multi-nanoheterostructures [16-18]. Of great interest is also reflected in research intraband optical transitions between quantum-states in multilayer spherical nanoheterostructures. Such attention to the above optoelectronic phenomena related to the possible creation on their basis of infrared detectors, high-speed modulators, and emitters cascade lasers [19,20].

In [21] carried out a theoretical study of the restructuring of the energy spectrum of charge carriers in quantized spherical layer under the action of a uniform electric field and the influence of the field on the form of the band intraband optical absorption.

Interest in the study of the electro-optic effects in quasizero-semiconductor systems is determined by the fact that in them the Stark shift of the energy levels of spatially confined electron-hole pairs (excitons) is not accompanied by a sharp drop in the oscillator strength of the corresponding transitions in the quantum dots, which has a large value, exceeding the typical values oscillator strength of the transitions in semiconductors [22]. As a result, the exciton states in electric fields, much higher than the ionization field in the bulk semiconductor, are not destroyed by shifts in excess of the value of the exciton binding energy [22].
In $[16,17,22]$ investigated the influence of the electric field intensity up $10^{7} \mathrm{BM}^{-1}$ on the absorption spectra of glasses doped with $\mathrm{CdS}$ nanocrystals and CdSSe, near the edge of the interband absorption. Found in [10] the dependence of the Stark shift of the energy levels of the electron and hole on the QD size was due to the peculiarities of the energy spectrum of a spatially bounded electron-hole pair (exciton) in a uniform external electric field. In $[16,17,22]$ not studied the question of the origin of the bulk of the excitons in the QD in an external uniform electric field. By means of the exciton-exciton volume, the structure of which (the Bohr radius and binding energy) is the same as in the exciton in a semiconductor single crystal [6-8].

In [10] developed a theory of the Stark effect in QDs in an environment where the polarization interaction of electrons and holes to the surface QD plays a dominant role [6-9]. It is found that the shifts of the energy levels of size quantization of electron-hole pair in a QD in an external uniform electric field in the region of interband absorption determined by the quantum-quadratic Stark effect. Proposed electro-optical method, enabling to determine the values of the critical radii QDs, which may arise bulk excitons [10].

Semiconductor QDs are used effectively in opto- and nanoelectronics. For example, IR laser have been fabricated on the basic of semiconductor QD heterostructures. Such devices have improved characteristics as compared with those of quantum well lasers. 
Recently, QDs found new applications in biology and medicine [1]. First of all, QDs are studied as new tools for obtaining an image of living cell and their diagnostics in vivo. As shown elsewhere [2-5], QDs have considerably better properties than fluorescent chromophores which are currently used in biology and medicine, namely: (i) higher quantum yield; (ii) optical activity in the long - wave range of the spectrum; (iii) and significantly greater photochemical stability.

Optical properties of QDs strongly depend on their dimensions. The emission wavelength is increased from $4 \cdot 10^{2}$ to $10^{3} \mathrm{~nm}$ with increasing the QD radius from 5,0 to $20,0 \mathrm{~nm}$ [6-8].

Unlike other fluorescent systems, semiconductor QDs have a threshold excitation energy; i.e., they can be excited by such and greater energies. Thus, the threshold energy must not be equal to the energy of transition within the QDs. Therefore, many QDs can be excited by the light with the same wavelength, whereas each QD emits the light with a different wavelength. Thus, unique spectral properties of QDs are very promising for biological and medicine studies.

In a number of papers (e.g., [3]) a study of multipurpose semiconductor QD samples for detection of cancer cells in living organisms and for creating images of these cell was described. A study of cancer cell growth in animals demonstrated that QDs were accumulated in tumors due to both improved transitivity and binding to the biomarker surface of cancer bodies according to the antibody mechanism. High - sensitivity color images of cancer cells have been obtained directly in living organism. Thus, new methods of obtaining high - sensitive images of molecular objects in a living organism using QDs were demonstrated.

Due to their narrow and symmetrical peaks in emitting spectra, semiconductor QDs can be used for optical diagnostics where different colors (wavelengths) and different intensities of the spectral peaks can be used for identification of genes, amino acids, proteins and the molecular chains.

This paper presents a new prospective method of amino acid diagnostics using semiconductor QDs. The main topic is an effect of the amino acid - QD interaction on the emission spectrum of QDs.

\section{Energy Shift of Quantum Dots in the Presence of Amino Acids}

It is known that all 20 amino acids fall into four groups: ones with negative charge; with positive charge; polar and nonpolar. Each amino acid type interacts with QDs in a different way and, thus, differently affects energy levels of charge carriers in QDs and, consequently, the QD emission spectrum. We have carried out detailed microscopic calculation of electron and hole spectra in QDs, which interact with charge amino acids $[4,5]$.
Let us consider an interaction of QD with charged amino acid (AA). For the sake of simplicity we shall approximate AA with a uniformly charge sphere. The electrical field on the surface of such sphere is:

$$
E=\frac{Q}{\varepsilon_{1} R^{2}} R
$$

where $\mathrm{R}$ is the sphere radius; $\mathrm{Q}$ is the charge, wherein $Q=(4 / 3) \pi \rho R^{3}$ and $\rho$ is the charge density; $\varepsilon_{1}$ is the dielectric constant.

Since the energy gap of QD is less than that of the surrounding material, the charge carrier motion in QD is confined by its volume. Thus, optical properties of QD are determined by the electronic structure of a spatially confined electron - hole pair (exciton) [6-15]. The QD AA interaction can be considered as effect of the electric field of AA on QD. The case is that under an external electric field a shift of dimensional quantization energy levels for an electron - hole pair in QD in the hand - to band absorption range is determined by the quadratic quantum - dimensional Stark effect [6-11].

As in $[9,10]$, let us consider a spherical QD with the radius $a$, the dielectric constant of QD being $\varepsilon_{2} \gg \varepsilon_{1}$. Let us study the effect of the electric field $E$ on the electron hole pair spectrum. The electron - hole pair Hamiltonian for QD exposed to the electric field includes, in addition to the kinetic energies of the electron and hole, the following: the energy of the Coulomb interaction of the electron and hole; the energy of the electron and hole interaction with their own images; the energies of interactions with "foreign" images, and the energy of electron and hole interaction with the field. The Hamiltonian can be significantly simplified when $\varepsilon_{2} \gg \varepsilon_{1}[6-9,11-15]$.

We shall consider a case when the QD dimension is confined by the condition:

$$
a_{h} \ll a \leq a_{e},
$$

where $a_{e}=\left(\varepsilon_{2} \hbar^{2} /\left(m_{e} e^{2}\right)\right) ; a_{h}=\left(\varepsilon_{2} \hbar^{2} /\left(m_{h} e^{2}\right)\right)$ are Bohr's radii of electron and hole, respectively; $e$ is the electron charge; $m_{e}$ and $m_{h}$ are the effective masses of electron and hole, respectively. If condition (2) is satisfied the polarization interaction provides the main contribution to the potential energy of electron and hole [6-8]. Furthermore, when $\left(m_{e} \ll m_{h}\right)$, condition (2) allows us to consider the heavy hole motion in the fields with the electron potential averaged over the total electron path (the so called adiabatic approximation) [6-8].

The electronic structure of an electron - hole pair within the adiabatic approximation can be easily calculated; see, for example [6-8]. After averaging the potential energy of the electron - hole pair over electron wave functions (in the case of a spherical potential well), we have an expression for the potential energy of a heavy hole moving in the adiabatic potential field of an electron $[9,10]$ :

$$
U\left(r_{h}, a, E\right)=\frac{\hbar^{2}}{2 m_{h} a_{h}^{2}}\left(\frac{a_{h}}{a}\right)\left[\frac{1}{1-\left(r_{h} / a\right)^{2}}+2 C i\left(2 \pi n_{e}\right)-2 C i\left(2 \pi n_{e}\left(r_{h} / a\right)\right)+\frac{\sin \left(2 \pi n_{e}\left(r_{h} / a\right)\right)}{2 \pi n_{e}\left(r_{h} / a\right)}+2 \ln \left(\frac{r_{h}}{a}\right)+\frac{\varepsilon_{2}}{\varepsilon_{1}}-4\right]-e E r,
$$


where $n_{e}$ is the electron principal quantum number; $r_{h}$ is the distance between the hole and the centre of QD; $C i(y)$ is the integral cosine. The last term in (3) results in shifting the potential well bottom for the hole in QD by a value $[9,10]$ :

$$
\Delta x=\frac{\left(\varepsilon_{2} / e\right)}{\left(1+(2 / 3) \pi^{2} n_{e}^{2}\right)} E a^{2}
$$

as well in shifting the dimensional quantization energy levels by a value:

$$
\Delta E_{h}=-\frac{\varepsilon_{2}}{2\left[1+(2 / 3) \pi^{2} n_{e}^{2}\right]} E^{2} a^{3},
$$

where $\Delta x=\left(r_{h} / a\right)$.

From (5) it follows that the energy shifts for electron and hole do not depend on the hole principal quantum number

$n_{h}$ and are determined solely by $n_{e}$. Under the electron field $E$ the sift of dimensional quantization energy levels $\Delta E_{c}$ will be about $[9,10]$ :

$$
\Delta E_{c} \approx \varepsilon_{2}\left(\frac{a_{e}}{a}\right)^{3} E^{2} a^{3} .
$$

For the ground electron state $n_{e}=1$ and if $\left(a_{e} / a\right) \leq$ (1/3), $\Delta E_{e} \ll \Delta E_{h}$. Thus, expression (5) describes the quantum - dimensional quadratic Stark effect, when quantum - dimensional energy levels shift under the electric field by a value proportional to $E^{2} a^{3}$. Figure 1 show an emission spectrum of InAs QD without and with an account for interaction with AA. For calculation, we took the following parameters of InAs: $a=5,0 \mathrm{~nm}, \varepsilon_{2}=14,9$, $Q=e, R=1,0 \mathrm{~nm}, \varepsilon_{2}=1,7$. The resulting shift of the emission peak was approximately $\approx 18 \mathrm{meV}$.

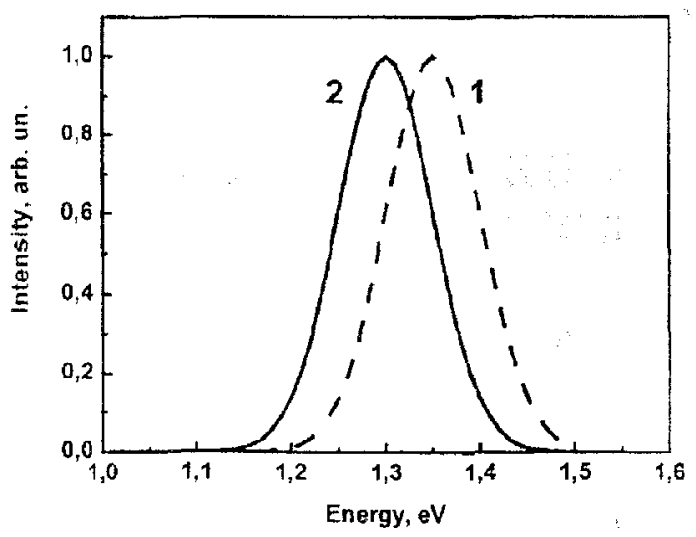

Fig 1. Emission spectrum of InAs $Q D$ without (curve 1) and with an account for interaction (curve 2) with charged $A A$.

\section{Comparison of Theory with Experiments}

In conclusion, we briefly discuss the possible experimental observations presented in $[9,10]$ of the new electro-optical method. In [6,7] investigated the interband absorption spectra dispersed in a transparent dielectric matrix bornosilikatnogo glass (with a dielectric constant $\varepsilon_{1}$ $=2.25$ ) nanocrystals $\mathrm{CdS}$ (with a dielectric constant $\varepsilon_{2}=9.3$ ) the size $a$ from 2 to $10^{2} \mathrm{~nm}$. The effective masses of electrons and holes in $\mathrm{CdS}$, respectively, and were equal $\left(\left(m_{e} / m_{0}\right)=0.205\right)$ and $\left.\left(m_{h} / m_{0}\right)=5\right)$. The theoretical spectrum of an exciton in a QD CdS, obtained in [6-8,1115], with good accuracy by the experimental dependence of the exciton spectrum of the radius and CT $[33,34]$ in the QD size $a$ not exceeding $10 \mathrm{~nm}$.

In experimental studies [6-8] observed peaks of enlightenment associated with transitions between levels of size quantization of the exciton in the transmission spectra of the nanocrystal CdSe (with a dielectric constant $\varepsilon_{2}=9.4$ ) radius $a=5 \mathrm{~nm}$, dispersed in a matrix of silica glass (with a dielectric constant $\left.\varepsilon_{1}=2.25\right)$. The effective masses of electrons and holes in CdSe QDs, respectively, and were equal $\left.\left(m_{e} / m_{0}\right)=0.13\right)$ and $\left.\left(m_{h} / m_{0}\right)=2.5\right)$.

In this case, the peak positions of enlightenment nanocrystals CdSe, as a function of radius $a \mathrm{QD}$, in the QD size $a=4.55 \mathrm{~nm}$ with good accuracy by the exciton spectrum [6-8,11-15]. Put quasi-zero system, studied under experimental conditions [6-8] in a uniform field of strength $F$. Consideration of the effect of the local field $[9,10]$ leads to the fact that the quasi-zero-systems on the QD acts field of strength

$$
F_{i n s}=f^{-1} F, \quad f=3 /\left[2+\left(\varepsilon_{2} / \varepsilon_{1}\right)\right],
$$

where the coefficient $f=0,489$ for CdS QDs and CdSe.

In [10] shows the values of the shifts $\Delta \lambda_{1,0,0}^{t_{h}}(a, F)$ levels of quantum hole in $\mathrm{CdS}$ and $\mathrm{CdSe}$ nanocrystals, respectively, in a uniform external electric field strength $F \leq 1,23 \cdot 10^{3} \mathrm{BM}^{-1}\left(F_{\text {ins }} \leq 6 \cdot 10^{2} \mathrm{BM}^{-1}\right)$

satisfying the conditions of applicability of are constructed theory [10].

In addition in [10], with increasing field strength $F$ is observed quadratic Stark effect, according to which the offset value $\Delta \lambda_{1,0,0}^{t_{h}}(a, F)$ and $\Delta V(F)$ increase $\left(\sim F^{2}\right)$. In this case, for CdS QDs with increasing $F$ of $1.0 \cdot 10^{3} \mathrm{BM}^{-1}$ to $1,23 \cdot 10^{3} B M^{-1}$ value of the Stark shift of the energy of the ground state bulk exciton $\Delta V(F)$ increases from $4,87 \cdot 10^{2} \mathrm{meV}$ to $7,37 \cdot 10^{2} \mathrm{meV}$, and for CdSe QDs with changing field strength $F$ of $2,1 \cdot 10^{2} \mathrm{BM}^{-1}$ to $5,1 \cdot 10^{2} \mathrm{BM}^{-1}$ the value $\Delta V(F)$ increases from $1,25 \cdot 10^{2} \mathrm{meV}$ to $7,78 \cdot 10^{2}$ meV.

Thus, it was found that the electro-optical properties of quasi-zero-systems containing semiconductor nanocrystals, in the interband absorption determined by the quantumquadratic Stark effect. A new electro-optical method, which gives the opportunity to determine the values of the critical radius of nanocrystals, which can be three-dimensional excitons $[9,10]$. 


\section{Conclusions}

A case was considered when polarization interaction of an electron and hole with the QD environment contributes mainly to rearrangement of the spectrum. It is shown that when QD interacts with charge amino acids, a shift of dimensional quantization energy levels for an electron hole pair in QD in the band - to - band absorption range is determined by the quadratic quantum - dimensional Stark effect. An analytical expression for the energy shift for electron and hole levels has been obtained as a function of the electron intensity and QD radius. A case was considered when dielectric constants of QDs and their environment are essentially different. This approach allows us to significantly simplify the expression for the polarized interaction energy of electron and hole with the QD environment.

The energy shift of electron and hole levels defining the shift of the QD luminescence pear has been calculated.

Thus, a good method for using semiconductor QDs for experimental detection of charged amino acid presence with high accuracy is provided.

Thus, it was found that the electro-optical properties of quasi-zero-systems containing semiconductor nanocrystals, in the interband absorption determined by the quantumquadratic Stark effect. A new electro-optical method, which gives the opportunity to determine the values of the critical radius of nanocrystals, which can be three-dimensional excitons $[9,10]$.

\section{References}

[1] C.W. Chan, Shuming Nie, Science. 281, 2016 (1998).

[2] X. Michalet, F.F. Pihaud, L.A. Bentolila, J.M. Tsay, S.Doose, J.J. Li, G. Sundaresan, A.M. Wu, S.S. Gambhir, S. Weiss, Science. 307, 538 (2005).
[3] X. Gao, Y. Cui, R. M. Levenson, L. W.K. Chung, S. Nie, Nature Biotechnology. 22, 969 (2004).

[4] A.S. Davydov, Biology and quantum mechanics. (Kiev: Naukova Dumka, 1979).

[5] Ch. P. Huang, T.M. Chen, K. Li, Biosensors and Bioelectronics. 6, 201 (2007).

[6] A.P. Spak, S.I. Pokutnyi, Progr. Phys. Metal. 6, 105 (2005).

[7] S.I. Pokutnyi, Ukr. J. Phys. Rev. 3, 46 (2006).

[8] S.I. Pokutnyi, Phys. Lett. A. 342, 347 (2005).

[9] S.I. Pokutnyi, Semiconductors. 34, 1079 (2000).

[10] S.I. Pokutnyi, J. Applied Phys. 96, 11115 (2004).

[11] A.P. Shpak, S.I. Pokutnyi, V. N. Uvarov, M.S. Pokutnyi, Metallophiz. Noveishie Tekhnol. 30, 459 (2008).

[12] S.I. Pokutnyi, Semiconductors. 46, 165 (2012).

[13] S.I. Pokutnyi, Phys. Express. 1, 158 (2011).

[14] S.I. Pokutnyi, J. Nanoscience Lett. 1, 191 (2011).

[15] S.I. Pokutnyi, Phys. Express. 2, 1 (2012).

[16] T. Wood, S. Burrus, Applied Phys. Lett. 44, 16 (1984).

[17] L. Jacak, A. Wojs, W. Nawrocka, Phys. Low-Dim. Structr. 3, 59 (2009).

[18] J.W. Haus, H.S. Honma, Phys. Rev. B. 57, 1359 (2009).

[19] R. Blick, R. Haug, Phys. Rev. B. 57, 7899 (2010).

[20] S. Schmitt-Rink, D. Miller, Phys. Rev. B. 56, 8113 (2009).

[21] J. Faist, F. Cappasso, D. Sinco, Science. 264, 553 (2004).

[22] K. Bajema, R. Marlin, Phys. Rev. B. 58, 1300 (2010). 